\title{
Dominant Sources of Variability in Passive Walking
}

\author{
Thrishantha Nanayakkara ${ }^{1}$, Katie Byl ${ }^{2}$, Hongbin Liu $^{1}$, Xiaojing Song ${ }^{1}$, Tim Villabona ${ }^{3}$
}

\begin{abstract}
This paper investigates possible sources of variability in the dynamics of legged locomotion, even in its most idealized form. The rimless wheel model is a seemingly deterministic legged dynamic system, popular within the legged locomotion community for understanding basic collision dynamics and energetics during passive phases of walking. Despite the simplicity of this legged model, however, experimental motion capture data recording the passive step-to-step dynamics of a rimless wheel down a constant-slope terrain actually demonstrate significant variability, providing strong evidence that stochasticity is an intrinsic-and thus unavoidable-property of legged locomotion that should be modeled with care when designing reliable walking machines. We present numerical comparisons of several hypotheses as to the dominant source(s) of this variability: 1) the initial distribution of the angular velocity, 2) the uneven profile of the leg lengths and 3) the distribution of the coefficients of friction and restitution across collisions. Our analysis shows that the 3rd hypothesis most accurately predicts the noise characteristics observed in our experimental data while the 1st hypothesis is also valid for certain contexts of terrain friction. These findings suggest that variability due to ground contact dynamics, and not simply due to geometric variations more typically modeled in terrain, is important in determining the stochasticity and resulting stability of walking robots. Although such ground contact variability might be an expected result in field robotics on significantly rough terrain, we again note our experimental data applies seemingly deterministic-looking terrains: our results suggest that stochastic ground collision models should play an important role in the analysis and optimization of dynamic performance and stability in robot walking.
\end{abstract}

\section{INTRODUCTION}

Although significant progress has been made in recent years in the field of robot locomotion, relatively few works to date have recognized that nondeterminism in ground contact dynamics can play a significant role in determining the overall stability and success of walking machines[1]. Unlike most other forms of locomotion - rolling or flying, for example - walking systems involve dynamics that are punctuated by impulsive ground-foot collisions, resulting in

*This work was partially supported by the Engineering and Physical Sciences Research Council, UK, under grant agreement EP/I028773/1, Radcliffe fellowship program, Harvard University, and the European Union 7th Framework Program, grant agreement 287728 (Project title: STIFFFLOP).

${ }^{1}$ Thrishantha Nanayakkara, Hongbin Liu, and Song Xiaojing are with the Division of Engineering, King's College, University of London, Room 2.47, Strand Building, Strand, London, WC2R 2LS, UK thrish.antha, hongbin.liu, song.xiaojing at kcl.ac.uk

2 Katie Byl is with the Electrical and Computer Engineering Dept., University of California, Santa Barbara, CA 93106, USA katiebyl at ece.ucsb.edu

${ }^{3}$ Tim Villabona is with the Research Laboratory of Electronics, Massachusetts Institute of Technology, 36-757, 50 Vassar St, Cambridge, MA 02139, USA timv at mit.edu near-instantaneous jumps in state space; predicting the variability of these "jumps" is essential in designing stabilizing control and in predicting the likelihood of falls. Recent work done on passive locomotion suggests that the secrets of efficient and stable walking lie in the coupled dynamics of the moving body and the ground, rather than the kinematics and dynamics of the moving body alone [2], [3]. Attempts to understand the subtle features of these dynamics using a simple passive walker known as the rimless wheel [6] show that variability across steps and trials is an intrinsic property of walking even in steady state walking on the same terrain [4] $-[8]$.

Experimental results of recent walking robots such as Whegs [9], Rhex [10], Mecharoach [11], and Hade [12] apparently prove that the trial to trial variability of each robot's behavior can not be explained by the neuromechanical models of their biological origins alone. Variability of walking is an indispensable property of walking [15] to deal with metastability of legged locomotion [7]. This evidence, as well as the results presented here, suggest that variability is an inevitable design consideration and is important to model and account for in legged locomotion.

Therefore, the focus of this paper is to characterize the variability of passive locomotion of a rimless wheel with rigid spokes that adequately represent the salient features of walking in the context discussed above. We present experimental evidence for variability in a seemingly deterministic environment, where the rimless wheel was repeatedly rolled down a smooth-surfaced, wooden ramp starting from a given location and posture. Then we made an open hypothesis as to several possible sources of variability for this system. Numerical results of simulations were used to quantify the potential of each hypothesis to explain the experimental phenomena. Moreover, we contend that variability determines an important portion of walking that can be effectively harnessed by understanding its dominant sources.

Rest of the paper is organized as follows. Section II discusses the derivation of discrete dynamics of the rimless wheel. Section III explains the experimental set up, instruments used, and the procedure to collect data. Section IV elaborates the experimental evidence of variability, the hypotheses on the source of variability and their experimental and numerical assessment. Finally, Section V gives a discussion and conclusion on the results as well as future directions of research. 


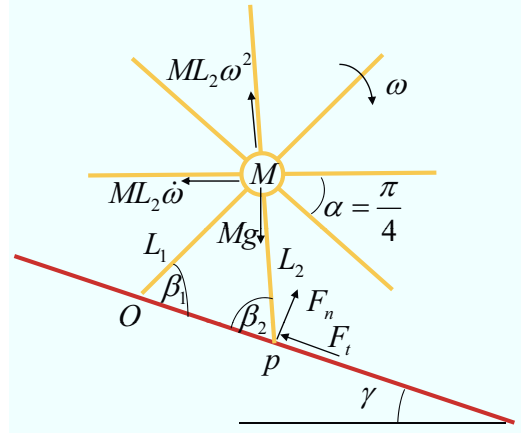

Fig. 1. The rimless wheel model with legs of zero mass and a hub of mass $M=1.39[\mathrm{~kg}]$. The lengths of the legs in contact with the ground at point $O$ and that collides on the ramp at point $p$ are $L_{1}$ and $L_{2}$ respectively. The angular speed of the rimless wheel is $\omega$, and the impulse and friction forces at point $p$ are $F_{n}$ and $F_{t}$ respectively. The two legs momentarily in contact with the slope at points $O$ and $p$ make $\beta_{1}$ and $\beta_{2}$ angles with the ramp respectively. Lengths of the eight legs were measured to be $0.1524[\mathrm{~m}]$, 0.1524 [m], 0.1514 [m], 0.1514 [m], 0.1516 [m], 0.1508 [m], 0.1524 [m], and $0.1528[\mathrm{~m}]$. The eight legs are equally spaced with an angle $\alpha=\pi / 4$.

\section{The Discrete Dynamic Model of The Rimless WHEEL}

The rimless wheel as a dynamic system to study passive dynamic locomotion was introduced by Mcgeer [14]. The primary assumptions are point mass at the hip, no slip, inelastic impulsive collisions, and instantaneous transfer of support at collision (no double support, no aerial phase). Many of these assumptions are violated in our experiment; it is interesting to understand which assumptions are least reasonable.

Fig. 1 shows the basic kinematics and the forces acting on the rimless wheel when it is in passive locomotion: i.e., input energy derived from gravity alone. Passive locomotion provides an open loop control scenario to study the dynamics of interaction between the legs and the ground. In addition, the rimless wheel provides the most basic and essential features of walking, in that it samples a discrete set of ground contact points from an infinite set of points on the ground profile, and it loses kinetic energy in each collision with the ground.

\section{A. Collision dynamics of the rimless wheel}

In Fig. 1, let the mass of the hub be $M$ and the mass of a rod passing through the hub be $m$ respectively such that $M>>m$.

Fig. 2 shows the three stages we consider to derive the dynamics in the form of a return map across collisions.

At collision between $t=t_{1}^{-}$and $t=t_{1}^{+}$, the angular velocity drops both due to restitution and friction at the contact surface [14] [16]. Therefore the kinetic energy immediately after the collision is given by,

$$
\frac{1}{2} I\left(\omega_{1}^{+}\right)^{2}=\frac{1}{2} I\left(\omega_{1}^{-}\right)^{2}-\epsilon
$$

where, $\epsilon$ is the net energy loss at $t=t_{1}^{-}$. The net energy loss due to collision consist of three components: 1) loss during the compression and restitution phases of the collision, 2)

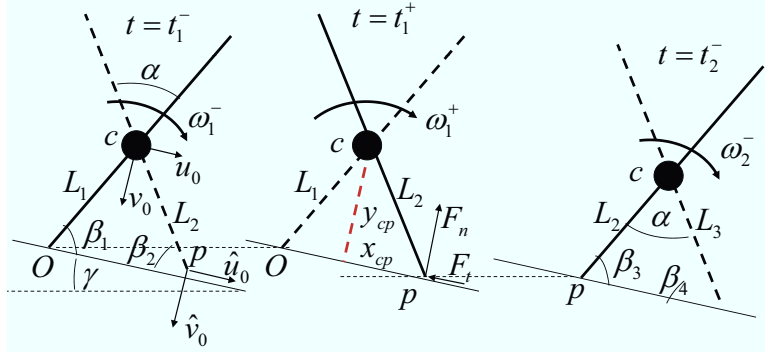

Fig. 2. The key stages between two consecutive collisions. The leg of length $L_{2}$ is about to collide on the ramp at point $p$ with a normal speed $\hat{v}_{0}$, tangential speed $\hat{u}_{0}$ and angular speed $\omega_{1}^{-}$at time $t=t_{1}^{-}$. An impulsive normal force $F_{n}$ and tangential force $F_{t}$ are generated immediately after the collision at time $t=t_{1}^{+}$that changes angular speed from $\omega_{1}^{-}$to $\omega_{1}^{+}$. The normal distance between the center $c$ and contact point $p$ is denoted by $y_{c p}$ and that along the slope is denoted by $x_{c p}$. The next leg of length $L_{3}$ approaches collision at time $t=t_{2}^{-}$with an angular speed $\omega_{2}^{-}$. The difference between $\omega_{2}^{-}$and $\omega_{1}^{+}$is caused by compression, restitution, slippage, and change of potential energy.

friction loss due to slipping during collision, and 3) potential energy loss/gain due to slipping up/down the ramp. The momentum normal to the slope causes compression and restitution at the contact point, and that tangential to the slope causes slipping. Let the normal and tangential velocities of the point $p$ just before colliding with the slope in figure 2 be $\hat{v}_{0}$ and $\hat{u}_{0}$ and those for the center of gravity $c$ be $\hat{v}_{0}^{c}$ and $\hat{u}_{0}^{c}$. For the rimless wheel we consider, $v_{0}$ and $u_{0}$ are given by,

$$
\begin{aligned}
& v_{0}=-L_{1} \omega_{1}^{-} \cos (5 \pi / 8) \\
& u_{0}=L_{1} \omega_{1}^{-} \sin (5 \pi / 8)
\end{aligned}
$$

The normal speed $\hat{v}_{0}$, and tangential speed $\hat{u}_{0}$ at point $p$ are given by

$$
\begin{aligned}
& \hat{v}_{0}=\hat{v}_{0}^{c}+L_{2} \omega_{1}^{-} \cos \left(\beta_{2}\right) \\
& \hat{u}_{0}=\hat{u}_{0}^{c}-L_{2} \omega_{1}^{-} \sin \left(\beta_{2}\right)
\end{aligned}
$$

For a given magnitude of impulse $P$, the velocity normal to the slope $\hat{v}$ and that tangential to the slope $\hat{u}$ at point $p$ at any given time during collision are given by

$$
\begin{aligned}
& \hat{v}=\hat{v}_{0}-P / m_{j} \\
& \hat{u}=\hat{u}_{0}-j \mu P / n_{j}
\end{aligned}
$$

where, $j=1$ if point $p$ slips in the direction of $\hat{u}_{0}$ and $j=-1$ otherwise, $\mu$ is the coefficient of friction at the contact point, $m_{j}$ is the effective mass normal to the slope, and $n_{j}$ is that tangential to the slope [16] given by

$$
\begin{aligned}
m_{j} & =M r_{g}^{2} /\left(r_{g}^{2}+x_{c p}^{2}+j \mu x_{c p} y_{c p}\right) \\
n_{j} & =M r_{g}^{2} /\left(r_{g}^{2}+y_{c p}^{2}+j \frac{x_{c p} y_{c p}}{\mu}\right)
\end{aligned}
$$

where $M$ is the mass of the hub, $r_{g}=L_{2}$ is the radius of gyration of the leg in contact with the slope, $y_{c p}$ is the distance between the center $c$ and contact point $p$ normal to the ramp, and $x_{c p}$ is that along the slope. 
Here, we follow the definitions given in [16] to derive the energy loss during collision. In [16], the total impulse $P$ at collision is divided into two parts: $\bar{P}$ is that part acting during the compression phase, also known as the characteristic impulse, and $\eta \bar{P}$ is that part acting during restitution. Therefore the total impulse $P=(1+\eta) \bar{P}$. We can obtain $\bar{P}$ by setting $\hat{v}=0$ to mark the end of compression and substituting $m_{j}$ from equation (5) in equation (4). Using the notations $(\cdot)_{+}$for the case of $u_{0}>0$ and $(\cdot)_{-}$for $u_{0}<0$, we can write the following set of equations for the characteristic impulse.

$$
\begin{aligned}
\bar{P}_{+} & =\hat{v}_{0} m_{j} \\
& =\hat{v}_{0} M r_{g}^{2} /\left(r_{g}^{2}+x_{c p}^{2}+\mu x_{c p} y_{c p}\right) \\
\bar{P}_{-} & =\hat{v}_{0} m_{j} \\
& =\hat{v}_{0} M r_{g}^{2} /\left(r_{g}^{2}+x_{c p}^{2}-\mu x_{c p} y_{c p}\right)
\end{aligned}
$$

Rewriting equations in (4) using $\bar{P}_{+}$in equation (6), we obtain

$$
\begin{aligned}
\hat{v} & =\hat{v}_{0}\left(1-P / \bar{P}_{+}\right) \\
\hat{u} & =\hat{u}_{0}-\mu \hat{v}_{0} m_{j} P / n_{j} \bar{P}_{+}
\end{aligned}
$$

It is useful to know the part of the characteristic impulse $\bar{P}$ that stops slip. Let this proportion be denoted by $\zeta$. By setting $P=\bar{P}$ in 8 , we obtain the slip velocity at the end of compression $\hat{u}_{\text {comp }}$ given by

$$
\hat{u}_{\text {comp }}=\hat{u}_{0}\left(1-\mu \hat{v}_{0} m_{j} / n_{j}\right)
$$

Assuming tangential velocity drops linearly due to friction, and using the fact that $\hat{u}=0$ when slip stops, the constant $\zeta$ is given by

$$
\begin{aligned}
\zeta & =\frac{\hat{u}_{0}-0}{\hat{u}_{0}-\hat{u}_{\text {comp }}} \\
& =\frac{\hat{u}_{0} n_{j}}{j \mu \hat{v}_{0} m_{j}}
\end{aligned}
$$

Therefore, the $\zeta \bar{P}$ component of the characteristic impulse $\bar{P}$ stops slip and the rest of the impulse works during slip reversal. By substituting $\bar{P}_{-}$from 7 and $\zeta$ from equation (10) in equation (4), we can obtain the corresponding velocities at the point of contact $p$ during slip reversal, as given by

$$
\begin{aligned}
& \hat{v}=\hat{v}_{0}\left(1-\zeta-\left(P-\zeta \bar{P}_{+}\right) / \bar{P}_{-}\right) \\
& \hat{u}=\mu^{2} \hat{v}_{0} m_{-j}\left(P-\zeta \bar{P}_{+}\right) / n_{-j} \bar{P}_{-}
\end{aligned}
$$

With these preliminary definitions, we can now proceed to derive some of the intermediate results not presented in [16]. We use the internal dissipation hypothesis in [16] to derive the equations for energy loss during compression, restitution, slip in the direction of $\hat{u}_{0}$, and slip reversal, since it has been proved in [16] to be the only hypothesis among several others like the kinematic hypothesis and impulse hypothesis, that gives a positive energy dissipation during both slip and slip reversal.

The internal dissipation hypothesis states that the square of coefficient of restitution $\eta^{2}$ is the ratio of elastic strain energy released at the contact point during restitution to the energy absorbed by deformation during compression.

Following the notation in [16] that part of the impulse force working after slip stops is denoted by $(\tau-\zeta) \bar{P}_{-}$, so that slip stops during compression if $0<\zeta<1$ and during restitution if $1<\zeta<\tau$. The limit $\tau$ of $\zeta$ marks the end of restitution.

We derive the normal and tangential energy loss in two parts: the first part from collision to slip stop, and the second part during slip reversal.

Let $D_{p r e}^{n}$ denote the energy lost in the direction normal to the slope before the slip stops. Then

$$
D_{\text {pre }}^{n}=\int_{P=0}^{P=\zeta \bar{P}} \hat{v} d P
$$

Substituting $\hat{v}$ from equation (8) in equation (12),

$$
\begin{aligned}
D_{\text {pre }}^{n} & =\int_{P=0}^{P=\zeta \bar{P}_{+}} \hat{v}_{0}\left(1-P / \bar{P}_{+}\right) d P \\
& =\hat{v}_{0}\left(\zeta \bar{P}_{+}-\frac{1}{2} \zeta^{2} \bar{P}_{+}\right) \\
& =\frac{1}{2} \hat{v}_{0} \zeta \bar{P}_{+}(2-\zeta)
\end{aligned}
$$

Let $D_{p r e}^{t}$ denote the energy lost due to slipping in the direction of $\hat{u}_{0}$. Then

$$
D_{\text {pre }}^{t}=\int_{P=0}^{P=\zeta \bar{P}} \hat{u} \mu d P
$$

Substituting $\hat{u}$ from equation (8) in equation (14),

$$
\begin{aligned}
D_{\text {pre }}^{t} & =\int_{P=0}^{P=\zeta \bar{P}_{+}}\left(\hat{u}_{0}-\frac{\hat{v}_{0} \mu m_{j} P}{n_{j} \bar{P}_{+}}\right) \mu d P \\
& =\mu \hat{u}_{0} \zeta \bar{P}_{+}-\left(\frac{\hat{v}_{0} \mu m_{j} \zeta \bar{P}_{+}}{2 \hat{u}_{0} n_{j} \bar{P}_{+}}\right) \mu \zeta \bar{P}_{+}
\end{aligned}
$$

Since $\zeta=\frac{\hat{u}_{0} n_{j}}{j \mu \hat{v}_{0} m_{j}}$ in equation (10), $\frac{\hat{v}_{0} \mu m_{j} \zeta}{\hat{u}_{0} n_{j}}=1$ in equation (15). Therefore we obtain,

$$
D_{\text {pre }}^{t}=\frac{1}{2} \mu \zeta \hat{u}_{0} \bar{P}_{+}
$$

Similarly, the energy loss in the direction normal to the slope for impulse force from $\zeta \bar{P}$ to the terminal value of impulse $P=P_{f}$ is given by

$$
D_{\text {post }}^{n}=\int_{P=\zeta \bar{P}}^{P=P_{f}} \hat{v} d P
$$

In [16] the terminal value of impulse $P_{f}$ is defined to be $P_{f}=\zeta \bar{P}+(\tau-\zeta) \bar{P}_{-}$, where $\tau$ is defined for various ranges of $\zeta$ as given by

$$
\tau= \begin{cases}1+\eta \sqrt{(1-\zeta)^{2}+\zeta(2-\zeta) \bar{P}_{+}} & \text {if } \zeta \leq 1 \\ 1+\sqrt{(\zeta-1)^{2}\left(1-\frac{\bar{P}_{+}}{P_{-}}\right)+\frac{\eta^{2} \bar{P}_{+}}{P_{-}}} & 1<\zeta<1+\eta \\ 1+\eta & 1+\eta<\zeta\end{cases}
$$


By substituting $\hat{v}$ from 11 and $P_{f}=\zeta \bar{P}+(\tau-\zeta) \bar{P}_{-}$in 17 , we obtain

$$
\begin{aligned}
D_{\text {post }}^{n} & =\int_{P=\zeta \bar{P}}^{P=P_{f}} \hat{v}_{0}\left(1-\zeta-\frac{P-\zeta \bar{P}_{+}}{\bar{P}_{-}}\right) d P \\
& =\frac{1}{2} \hat{v}_{0}(\tau-\zeta) \bar{P}_{-}(2-\zeta-\tau)
\end{aligned}
$$

The corresponding energy loss due to slip reversal is given by

$$
D_{\text {post }}^{t}=\int_{P=\zeta \bar{P}}^{P=P_{f}} \hat{u} \mu d P
$$

Substituting $\hat{u}$ from equation (11) in equation (20) we obtain,

$$
\begin{aligned}
D_{\text {post }}^{t} & =\int_{P=\zeta \bar{P}}^{P=P_{f}} \frac{\mu^{2} \hat{v}_{0} m_{-j}\left(P-\zeta \bar{P}_{+}\right)}{n_{-j} \bar{P}_{-}} d P \\
& =\frac{1}{2} \frac{\hat{v}_{0} \mu^{2} m_{-j}}{n_{-j}}(\tau-\zeta)^{2} \bar{P}_{-}
\end{aligned}
$$

Therefore the total energy lost in the tangential and normal directions of the slope are given by,

$$
\epsilon_{t}=D_{\text {pre }}^{t}+D_{\text {post }}^{t}
$$

and

$$
\epsilon_{n}=D_{\text {pre }}^{n}+D_{\text {post }}^{n}
$$

During experiments, we observed that the rimless wheel sometimes reverses the slip direction. This causes the rimless wheel to gain or lose potential energy during slipping downwards or upwards the ramp. In order to calculate the distance slipped, we should know the time spent on slipping in the direction of $\hat{u}_{0}$ or in the opposite direction. We derive the total time for compression and restitution by assuming that the ground can be represented by the Kelvin-Voigt (K-V) viscoelastic model given by,

$$
F=K_{r} d+C_{r} \dot{d}
$$

where $K_{r}$ and $C_{r}$ are the elastic coefficient and damping coefficient of the ramp, and $d$ is the amount of deformation of the ramp at contact point $p$ at any given time. The resulting motion equation is:

$$
\ddot{d}+2 \xi \omega_{n} \dot{d}+\omega_{n}^{2} d=0
$$

where, the undamped natural frequency $\omega_{n}=\sqrt{K_{r} / m}$ and the damping ratio $\xi=C_{r} /\left(2 \sqrt{K_{r} m}\right)$. The damped natural frequency is thus:

$$
\omega_{d}=\omega_{n} \sqrt{1-\xi^{2}}=\sqrt{\left(K_{r} / m\right)\left(1-\xi^{2}\right)}
$$

Assuming the impact duration is half-period of vibration, the coefficient of the restitution coefficient $\eta$ can be computed as [17]:

$$
\eta=e^{-\frac{\xi \pi}{\sqrt{1-\xi^{2}}}}
$$

and the time to release (one compression-restitution cycle) is $T_{r}=\pi / \omega_{d}$. Since the coefficient of restitution $\eta$ is sampled from a known distribution, and the elastic coefficient $K_{r}=$ $9000[\mathrm{~N} / \mathrm{m}]$ is known for standard lumber [18], the natural frequency $\omega_{d}$ can be readily computed using equations (26) and (27).

The time from the beginning of collision to slip stop $t_{p}$ is

$$
t_{p}= \begin{cases}t_{r} \zeta & \text { if } \zeta \leq 1 \\ t_{r}+t_{r}\left(\frac{\zeta-1}{\eta}\right) & \text { if } 1<\zeta<1+\eta \\ 1+\eta \text { if } 1+\eta<\zeta & \end{cases}
$$

Thus the duration from slip stop to the end of collision $T_{n}=T_{r}-T_{p}$. Since the energy lost due to friction is known (equations (16) and (21)), and the fact that $\zeta \bar{P}_{+}$part of the impulse acts till slip stops and $(\tau-\zeta) \bar{P}_{-}$part of the impulse acts during slip reversal, the slip distances are given by

$$
\begin{aligned}
S_{+} & =\frac{D_{p r e}^{t} T_{p}}{\zeta \bar{P}_{+}} \\
S_{-} & =\frac{D_{p o s t}^{t} T_{n}}{(\tau-\zeta) \bar{P}_{-}}
\end{aligned}
$$

The net slip in the direction of $\hat{u}_{0}$ is given by

$$
S=S_{+}-S_{-}
$$

Therefore the potential energy gained due to slipping is given by

$$
\epsilon_{p o t}=M g S \sin (\gamma)
$$

Therefore, the net energy lost during collision is given by

$$
\epsilon=\epsilon_{n}+\epsilon_{t}-\epsilon_{p o t}
$$

where $\epsilon_{n}$ is the energy loss due to movement normal to the ramp and $\epsilon_{t}$ is that due to slipping along the ramp, $\epsilon_{p o t}$ is the potential energy gained due to slipping along the ramp. Applying energy conservation law to states at $t=t_{1}^{+}$and $t=t_{2}^{-}$

$$
\begin{aligned}
\frac{1}{2} I\left(\omega_{2}^{-}\right)^{2} & =\frac{1}{2} I\left(\omega_{1}^{-}\right)^{2} \\
& +(2 M g L \sin (\gamma) \cos (\beta)-\epsilon) \\
\left(\omega_{2}^{-}\right)^{2} & =\left(\omega_{1}^{-}\right)^{2} \\
& +\frac{2}{I}(2 M g L \sin (\gamma) \cos (\beta)-\epsilon)
\end{aligned}
$$

where,

$$
\begin{aligned}
\beta & =\sin ^{-1}\left[\frac{L \sin (\alpha)}{\hat{L}}\right] \\
\hat{L} & =\sqrt{2} L \sqrt{(1-\cos (\alpha))}
\end{aligned}
$$

This can be written in the form

$$
x_{k+1}=x_{k}+\psi
$$

where state $x_{k}=\left(\omega_{1}^{-}\right)^{2}$ measured just before the $k$ th collision to $x_{k+1}=\left(\omega_{2}^{-}\right)^{2}, \psi=\frac{2}{I}(2 m g L \sin (\gamma) \cos (\beta)-\epsilon)$ is the net energy gain/loss due to gravity/friction and restitution. 


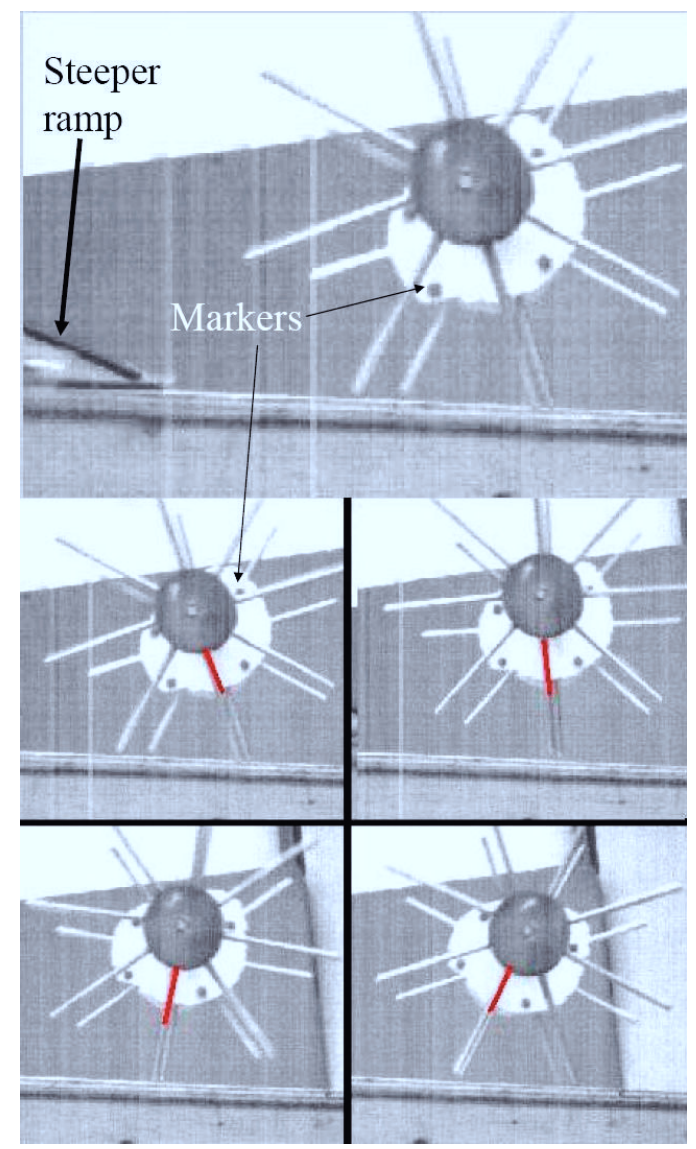

Fig. 3. The experimental set up. The rimless wheel was released at rest on the steeper ramp so that it collides only once on it before colliding on the longer ramp.

\section{EXPERIMENTAL PROCEDURE}

The fabricated rimless wheel consists of an aluminum body with radial legs. Eight legs of mean length $0.203 \mathrm{~m}$ protrude out of a central hub of mass $1.39 \mathrm{~kg}$. The individual lengths were $[0.1524,0.1524,0.1514,0.1514,0.1516$, $0.1508,0.1524,0.1528] \mathrm{m}$. To fit the experimental procedure to two-dimensional space, we constructed two rimless wheels, rigidly bounded by an axle. We constructed a ramp of $9.59^{\circ}$ slope and $3.66 \mathrm{~m}$ length, out of standard construction lumber. All forms of the experiment consisted of the rimless wheel rolling down this ramp, with motion capture markers affixed to the rimless wheel's central hub. Three experiments were done. In the first one, 51 trials were conducted by rolling the rimless wheel from a steeper ramp of $30.59^{\circ}$ as shown in Fig. 3. In each trial, the rimless wheel was held by the same leg, at a marked location on the steeper ramp and it was released at zero velocity allowing it to gain angular velocity by colliding once on the steeper ramp before entering the second ramp of $9.59^{\circ}$. We conducted another 48 trials by releasing the rimless wheel from the less steeper ramp itself. This method allowed for clearer observation of the convergence properties of rimless wheel to its steady state starting from two different distributions of initial velocity. The third experiment was done on similar

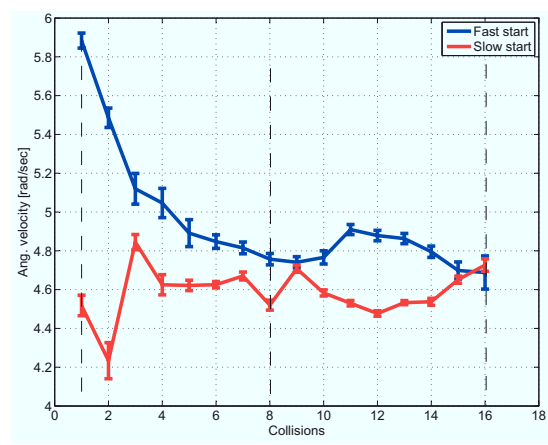

Fig. 4. Experimental results: the average velocity profiles of the rimless wheel measured at each collision on the ramp with error bars. The slope of the was ramp $9.59^{\circ}$. Fast start experiment had a starting velocity distribution $N\left(5.83,0.28^{2}\right) \quad[\mathrm{rad} / \mathrm{sec}]$, and that of the slow start experiment was $N\left(4.51,0.36^{2}\right)[\mathrm{rad} / \mathrm{sec}]$. The vertical dashed lines mark the period of the wheel where the first leg collides the slope again.

conditions of the first experiment with the only difference being the slope of the longer ramp was $7.02^{\circ}$. The reduced slope helped us to obtain intermittent failure states where the rimless wheel came to rest on the ramp allowing us to study the metastability properties of the rimless wheel.

We used an algorithm provided in [13] along with thirddegree Savitzky-Golay smoothing to obtain the angular displacement of the rimless wheel from the 3-D x-y-z coordinates provided by the motion-capture system at 120 frames per second resolution.

The rimless wheel drops the angular speed whenever a leg collides with the ground. This is true for collisions as well as bounces in between collisions. We make use of two additional physical realities to do this. One is the fact that the rimless wheel gains kinetic energy due to fall under gravity till a collision occurs. The other is the fact that true collisions should be separated by $45^{\circ}$ angles. Therefore, first we detect all peak angular velocities that rise at least $0.4[\mathrm{rad} / \mathrm{sec}]$ above the two sides. Then, we mark the first peak corresponding to the first collision with the ground, and look for the next velocity peak closest to a $45^{\circ}$ angle of rotation of the rimless wheel. We repeat this process for the first 16 collisions.

\section{EXPERIMENTAL EVIDENCE OF VARIABILITY}

Fig. 4 shows the average angular velocity profiles of the rimless wheel measured at each leg contact on the ramp of $9.59^{\circ}$ slope, with corresponding error bars (size of an error bar represent the standard error across all trials). We can observe from Fig. 4 that the size of the error bars along both average speed profiles tend to reduce across collisions. However, even in the steady state, the variability does not vanish completely. For instance the error bars tend to increase toward the latter part. Careful observation of the experimental data showed that slipping along the ramp toward the latter part of the trial do play an important role in the variability of speed. It also suggests that a single trial carries limited information about the overall dynamics of legged locomotion. This is further illustrated in Fig. 5, 

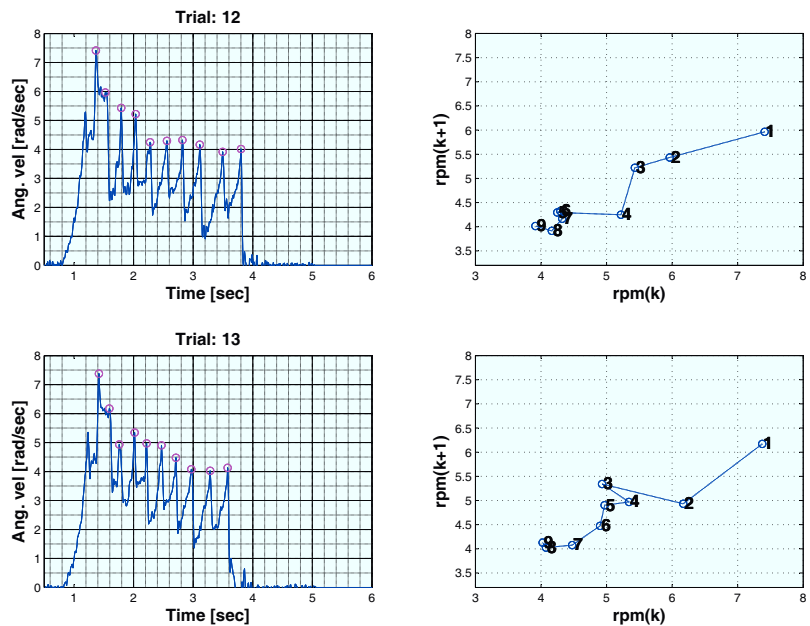

Fig. 5. Two consecutive trials picked in random.

where two consecutive trials from the experiment on $7.02^{\circ}$ ramp differ in detail from one another but share some overall pattern. The first and third plots are the angular velocity profiles of the two consecutive trials. The first collision was omitted because it was made on a steeper ramp to gather momentum. The second and fourth plots show the return maps for the respective trials. It is clear from these return maps that the details of the convergence pattern to a steady state are different for the two trials.

Before going into deeper investigations into a discussion on variability of passive locomotion of the rimless wheel, let us re-visit the return map shown in (37). The nature of the return map shown in (37) is that state vector $x_{k}$ is iterated by the function $x_{k+1}=f\left(x_{k}, \psi, \eta\right)$ without any external control. Stability and steady state behavior depends on the balance between the energy injected by gravity and that lost at collisions. We make three open hypotheses on the source of variability of dynamic walking: For a given distribution of the initial velocity, the subsequent variability of the angular velocities at collisions predominantly depends on

Hypothesis-1: the initial distribution of angular velocity.

Hypothesis-2: the uneven profile of leg lengths.

Hypothesis-3: the distribution of the coefficient of restitution and friction at each collision.

\section{A. Effect of initial distribution of angular velocity and the uneven profile of leg lengths}

In figure 4 both velocity profiles converge to the same velocity distribution with $(P>0.07$, standard two sided ttest) beyond the 12th collision. For the sake of clarity, the value of $P$ in statistical comparison between data sets of two random variables indicate the probability that the two data sets are coming from the same distribution. We generally conclude that the two data sets do not come from the same distribution if $P<0.05$. Therefore, the initial distribution of velocities (in this case, $N\left(5.83,0.28^{2}\right)[\mathrm{rad} / \mathrm{sec}]$ for the fast start experiment, and $\left.N\left(4.51,0.36^{2}\right)\right)[\mathrm{rad} / \mathrm{sec}]$ for the slow start experiment) alone can not be a determining factor in the steady state variability. We conducted another
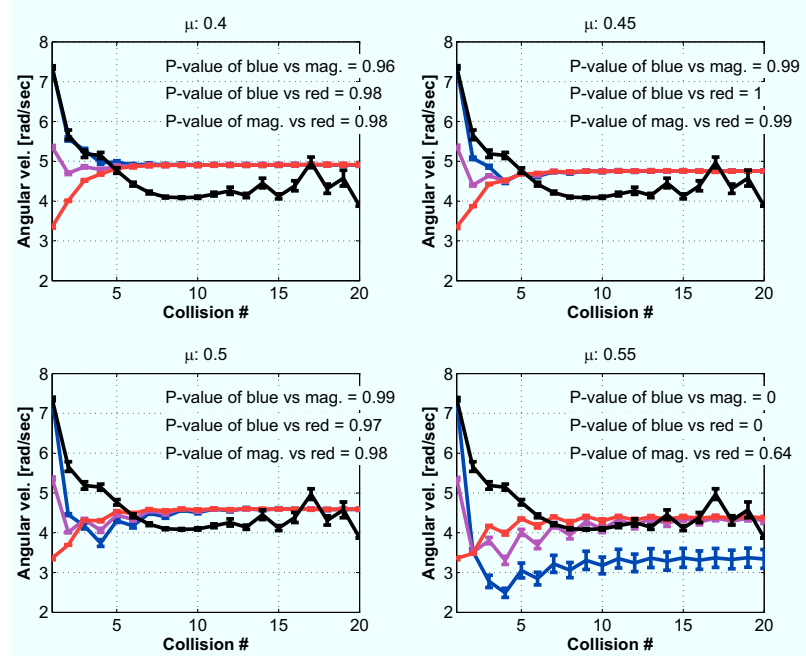

Fig. 6. Experimental and simulated velocity profiles for three differen mean start velocity profiles given by $N\left(1.35+2 k, 0.198^{2}\right), k=1,2,3$ by keeping $\eta=0.4$. $k=1,2,3$ correspond to "red", " "magenta", and " "blue" lines respectively. The $P$ values are given for each combination of statistical comparisons (blue vs red, red vs magenta, blue vs magenta) of steady state velocities ( $>12$ th collision).

set of 50 trials on a lower ramp angle with $7.02^{\circ}$ slope to obtain a longer steady state $(>12$ th collision) so that we can better understand the effect of the initial velocity distribution on the steady state variability of the rimless wheel. We compared numerical simulation results for three initial velocity distributions given by $N\left(1.35+2 k, 0.198^{2}\right)$, $k=1,2,3$ with the experimental measurements, so that the experimental initial velocity distribution is identical to that of simulation conditions when $k=3$ as shown in figure 6. Four cases were considered for $\mu=0.35+0.05 j, j=1,2,3,4$ to test if the effect of interaction between the initial velocity and the friction has an effect on the steady state behavior. The distribution of the coefficient of restitution was fixed at $\eta=$ $N\left(0.4,0.2^{2}\right)$ across all four cases with $1>\eta>0$, and the lengths of the legs were set to the experimentally measured values - $[0.1524,0.1524,0.1514,0.1514,0.1516,0.1508$, $0.1524,0.1528][\mathrm{m}]$. The velocity profiles corresponding to $\mu<0.5$ do not show a significant difference $(P>0.9$ for all combinations) in their the steady state variability. However, steady state velocity profile corresponding to $k=3$ (initial velocity distribution identical to that of the experiment) showed statistically significant difference $(P<0.001$ against both the "red" and "magenta" velocity profiles) for $\mu>0.5$. Moreover, we notice from the size of the error bars around the average velocity profile that the steady state variability increases with the increasing variability of $\mu$ and increasing initial velocity, suggesting that the interaction effect of $\mu$ and $\hat{u}_{0}$ in $D_{\text {pre }}^{t}$ and that between $\mu$ and $\hat{v}_{0} D_{\text {post }}^{t}$ should be an important design consideration.

In order to test whether the slight variability of the 

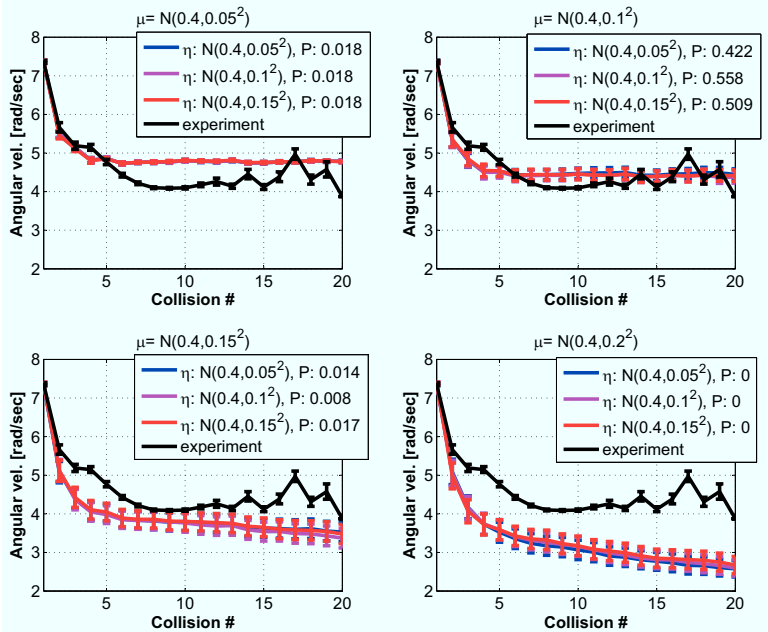

Fig. 7. Experimental and simulated velocity profiles for $\eta=$ $N\left(0.4,(0.05 k)^{2}\right), k=1,2,3$, for $\mu=N\left(0.4,(0.05 j)^{2}\right), j=$ $1,2,3,4$.

lengths of the legs $N\left(0.1519,0.000687^{2}\right)$ [m] significantly contributed to the steady state variability in the above simulations, we set the lengths of all legs to the experimentally measured average length 0.1519 [m] and ran the above simulations again. We did not find a significant difference $P>0.1$ for all initial velocity distributions across all $\mu$ values. This suggest that the slight variability of leg lengths is not a statistically significant determining factor.

\section{B. Effect of the distribution of kinetic energy loss at each collision}

Then further investigated the contribution of the distribution of $\eta$ and $\mu$ and their interactions in the variability of steady state velocity. We performed simulations using the above model given in (37) for three different distributions of $\eta$ given by $\eta=N\left(0.4,(0.05 k)^{2}\right), k=1,2,3$ subjected to $1>\eta>0$ for four different variances of $\mu$ given by $\mu=N\left(0.4,(0.05 j)^{2}\right), j=1,2,3,4$. The average value of $\mu$ was set to 0.4 because we noticed in figure 6 that the initial velocity distribution did not significantly affect the steady state variability for average $\mu<0.5$. Leg lengths of the model were set to their corresponding measured values of the real rimless wheel used for experiments. Under each distribution of $\eta$ for $k=1,2,3,1000$ trials were repeated subject to the same distribution of the initial angular velocity found in the experiments given by $N\left(7.35,0.19^{2}\right)[\mathrm{rad} / \mathrm{sec}]$, on the ramp of $7.02^{\circ}$ slope. Fig. 7 shows average experimental velocity profile with standard error bars at each collision (black) and three simulated velocity profiles for the three distributions of $\eta$ discussed above: blue $(k=1)$, magenta $(k=2)$, and red $(k=3)$. The statistical t-test between the steady state experimental velocity profile and those of the simulations corresponding to different distribution of $\eta$ shows that the $P$ values are sensitive to the variance of $\mu$ but not so to the variance of $\eta$. Therefore, this suggests that an interaction between the distribution of $\eta$ and that of $\mu$ play
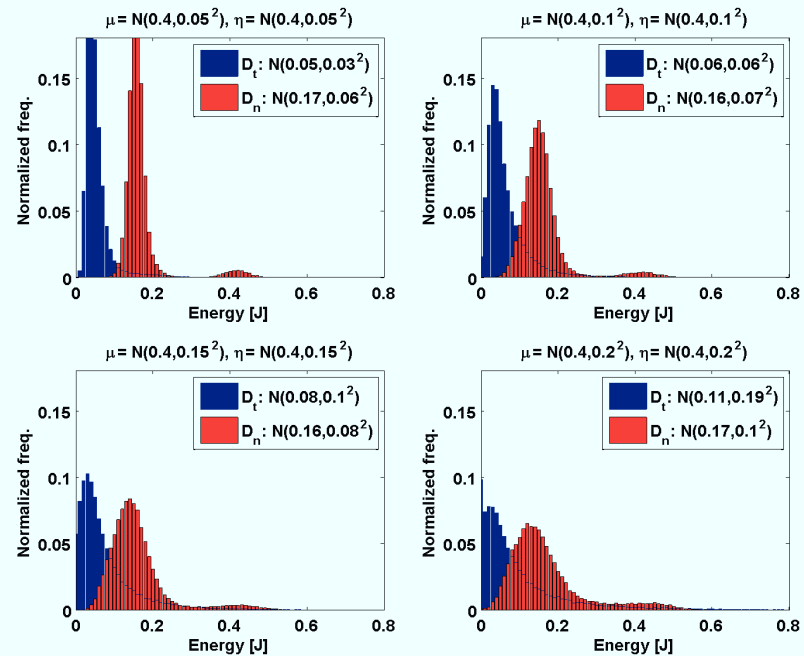

Fig. 8. The distributions of energy loss for movements along $\left(D_{t}\right)$ and normal $\left(D_{n}\right)$ to the ramp for $\mu=N\left(0.4,0.05 k^{2}\right)$ and $\eta=$ $N\left(0.4,0.05 k^{2}\right), k=1,2,3,4$.

a significant role in the variability of passive locomotion of the rimless wheel.

It is also evident in figure 8 that increased variance of $\mu$ increases both the average net energy lost along the tangential direction and its variance $\left(D_{t}\right.$ from $N\left(0.05,0.03^{2}\right)$ to $\left.N\left(0.11,0.19^{2}\right)\right)$. The net energy lost in the tangential direction is calculated by subtracting the potential energy gained by slipping down the slope from the energy lost due to friction (equations (22) and (33)). The increased variance of $\eta$ from $0.05^{2}$ to $0.2^{2}$ causes the variance of energy lost due to compression and restitution to increase from $0.06^{2}$ to $0.1^{2}$. However, it does not cause the average magnitude of energy lost normal to the ramp change much (stays around $0.17[\mathrm{~J}])$.

Then, we conducted another set of simulations for $\mu=$ $N\left(0.1+0.1 * n, 0.15^{2}\right), n=1,2,3,4$ and $\eta=N(0.1+$ $\left.0.2 * m, 0.15^{2}\right), m=1,2,3$ to test the effect of interaction between the average values of $\eta$ and $\mu$ on the steady state variability of the velocities. Figure 9 shows that the effect of interaction between the average values of $\mu$ and $\eta$. It is evident that the average value of $\eta$ influences the transient variability of velocity for low values of average $\mu(\mu<$ 0.3 ) with diminished effect on the steady state variability of velocity, and gradually increases its influence on the steady state variability of velocity for higher values of average $\mu$. The size of the error bars around average velocity profiles suggest that the average value of $\eta$ increases the steady state variability of velocity in general. However, this effect tend to reduce with the increase of average $\mu$. Overall, the combined effect of the magnitude of $\mu$ and $\eta$ show a nonlinear effect on both the transient and steady state variability of velocity.

\section{DISCUSSION AND CONCLUSIONS}

Despite considerable advances in robotic manipulation, elegant human-like walking still remains a distant goal. Lack of a deeper insight into what causes the variability of walking 

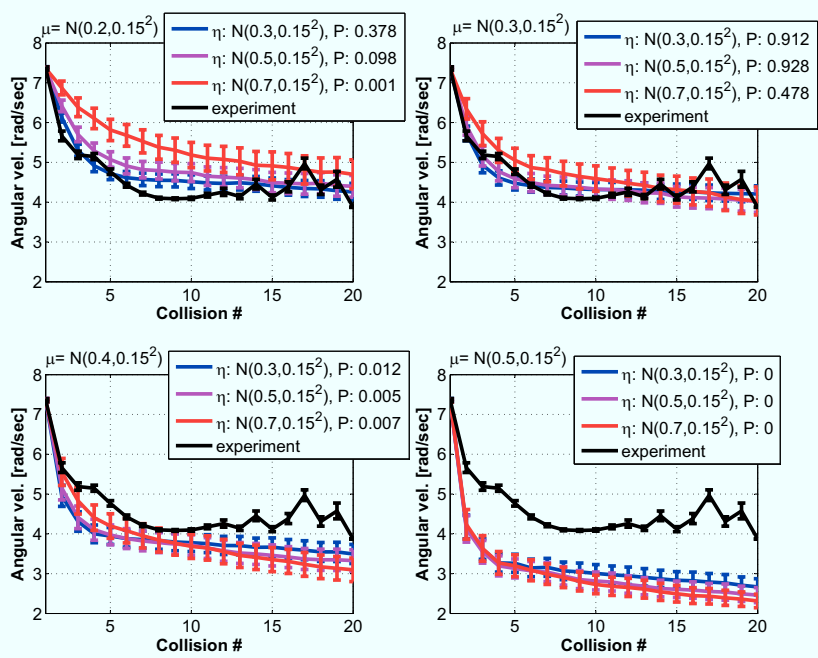

Fig. 9. Experimental and simulated velocity profiles for $\mu=N(0.1+$ $\left.0.1 n, 0.15^{2}\right), n=1,2,3,4$ and $\eta=N\left(0.1+0.2 m, 0.15^{2}\right), m=1,2,3$.

even on seemingly predictable flat terrains has been one of the key areas that needs further exploration to fill this gap. Passive dynamic walking provides a good open loop paradigm to study the effect of various causes and conditions of variability of walking because its only external input is gravity. This paper provides evidence that variability of walking is predominantly rooted in the distribution of friction and restitution across successive ground contact points of a passive dynamic walker on a smooth ramp. Moreover, we present evidence through numerical simulations, that the effect of the distribution of friction and restitution coefficients can be further modulated by the average starting velocity of the passive walker.

In this study, we presented experimental data and numerical simulations to suggest the foremost sources of variability of a passive dynamic walker on a smooth ramp. Of our three hypotheses-variability of initial velocity, coefficients of restitution and friction, and uneven leg lengths-we found that the coefficient of friction and restitution seem to play the most significant role, modulated by the magnitude of starting velocity. However, there are several other factors that should be examined in future works; things like bouncing, vibration [15], and exchange of static charges may also play a role in the emergence of variability.

The results presented in this paper not only sheds light on the predominant sources of variability of a passive dynamic walker, but also open various opportunities for research in areas like design of passive and active mechanisms of a robotic walker, variable adaptive control of walking ma- chines, machine learning and intelligent behavior of variable dynamic walkers, and so on. In conclusion, this paper suggests that a real time estimate of the distribution of friction and restitution coefficients across collisions will help a dynamic walker to predict the variability of walking.

\section{REFERENCES}

[1] R. L. Tedrake, "Applied optimal control for dynamically stable legged locomotion," PhD thesis, Massachusetts Institute of Technology, 2004

[2] S. H. Collins, A. Ruina, R. Tedrake, and M. Wisse, "Efficient Bipedal Robots Based on Passive Dynamic Walkers" Science, February 2005, vol. 307, pp. 1082 - 1085.

[3] J. Buchli, F. Iida, and R. Pfeifer, "Finding Resonance: Adaptive Frequency Oscillators for Dynamic legged Locomotion" in Proceedings of the IEEE/RSJ International Conference on Intelligent Robots and Systems (IROS 06), 2006, pp. 3903 - 3909.

[4] M. Garcia, A. Chatterjee, A. Ruina, and M. Coleman, "The Simplest Walking Model: Stability, Complexity, and Scaling" Journal of Biomechanical Engineering - Transactions of the ASME, April 1998, vol. 120, no. 2, pp. $281-288$.

[5] M. J. Coleman, A. Chatterjee, A. Ruina, "Motions of a Rimless Spoked Wheel: a Simplified 3D System with collisions", Dynamics and Stability of Systems, 1997, pp. $139-160$

[6] M. J. Coleman, "Dynamics and stability of a rimless spoked wheel: a simple 2D system with impacts", Dynamical Systems, vol. 25, Issue 2, pp. $215-238,2010$

[7] K. Byl and R. Tedrake, "Metastable Walking on Stochastically Rough Terrain”, in Proceedings of Robotics: Science and Systems IV, 2008

[8] M. H. Raibert, "Legged Robots that Balance", , MIT Press, Cambridge, MA; 1986.

[9] R. D. Quin, D. A. Kingsley, J. T. Offi, and R. E. Ritzmann, "Improved Mobility Through Abstracted Biological Principles", IEEE International Conference on Intelligent Robots and Systems (IROS 2002), pp. 2652-2657, 2002.

[10] U. Saranali, M. Buehler, and D. Koditscek, "Rhex a Simple and Highly Mobile Hexapod Robot", International Journal of Robotics Research, vol. 20, no. 7, pp. 616-631, 2001.

[11] M.J. Boggess, R.T. Schroer, R.D. Quinn, R.E. Ritzmann, "Mechanized Cockroach Footpaths Enable Cockroach-Like Mobility", Proceedings of the IEEE International Conference on Robotics and Automation (ICRA2004), vol. 3, pp. 2871 - 2876, 2004.

[12] E. Garcia, J. C. Arevalo, F. Sanchez, J. F. Sarria, and P. Gonzalez-deSantos, "Design and development of a biomimetic leg using hybrid actuators", Proceedings of the IEEE International Conference on Intelligent Robotics and Systems (IROS2011), pp. 1507 - 1512, 2011.

[13] S. D. Blostein, T. S. Huang, K. S. Arun, "Least-Squares fitting of two 3-D point sets", in IEEE Trans. on Pattern Analysis and Machine Intelligence, vol. 9, no. 5, pp. 698 - 700, September 1987.

[14] T. McGeer, "Passive dynamic walking", International Journal of Robotics Research, vol. 9, no. 2, pp. 62-82, 1990.

[15] T. McGeer and L. H. Palmer, "Wobbling, toppling, and forces of contact", American Journal of Physics, pp. 1089, vol. 57, Issue 12, Dec. 1989.

[16] W. J. Stronge, "Rigid Body Collisions with Friction", Proc. R. Soc. London, pp. 169-181, vol. 431, 1990.

[17] E. A. Butcher and D. J. Segalman, "Characterizing Damping and Restitution in Compliance Impacts via Modified K-V and HigherOrder Linear Viscoelastic Models", Journal of Applied Mechanics, vol. 67,pp. 831-834, 2000.

[18] J. M. Gere, and S. P. Timoshenko, 1990, Mechanics of Materials, 3rd edition, PWS-KENT Publishing Company, Elsevier Science BV, Amsterdam. 